\title{
Neural activity precedes conscious awareness of being in or out of a
}

\section{transient hallucinatory state}

1) Department of Biological and Medical Psychology, University of Bergen, Norway

2) Division of Psychiatry, Haukeland University Hospital, Bergen, Norway

3) Department of Radiology, Haukeland University Hospital, Bergen, Norway

4) NORMENT Center for the Study of Mental Disorders, Haukeland University Hospital, Bergen, Norway

5) Department of Clinical Engineering, Haukeland University Hospital, Bergen, Norway

6) Department of Clinical Medicine, University of Bergen, Norway

7) Department of Psychiatry and Medical Psychology, Medical University of Plovdiv, 4 Bulgaria

8) Department of Addiction Medicine, Haukeland University Hospital, Bergen, Norway

9) Department of Clinical psychology, University of Bergen, Norway

10) Rijks Universiteit Groningen (RUG), Department of Biomedical Sciences of cells and systems and Department of Psychiatry, University Medical Center Groningen (UMCG), 9 Netherlands

Corresponding author: Kenneth Hugdahl, University of Bergen, Norway, email: hugdahl@uib.no, phone: +47-55586277

The present study was funded by research grants from the European Research Council (ERC \# 693124), and Helse-Bergen (\#912045) to Kenneth Hugdahl, and by grants from the Medical 4 University of Plovdiv, Bulgaria Drozdstoy Stoyanov and by a TOP grant from ZonMW 5 (Dutch Medical Research Council) \# 91213009 to Iris E. Sommer,

6 Keywords: Schizophrenia, auditory hallucinations, fMRI, symptom-capture, onset, offset, 7 cingulate cortex

Acknowledgements: The authors want to acknowledge the contribution of MR-technicians 9 and research assistants, as well as the clinical and non-clinical participants, at each site. 
34 Summary

(203 words)

36 Auditory verbal hallucinations, or "hearing voices", is a remarkable state of the mind,

37 occurring in psychiatric and neurological patients, and in a significant minority of the general

38 population. An unexplained characteristic of this phenomenon is that it transiently fluctuates,

39 with coming and going of episodes with time. We monitored neural activity with BOLD-

40 fMRI second-by-second before and after participants indicated the start and end of a transient

41 hallucinatory episode during the scanning session by pressing a response-button. We show

42 that a region in the ventro-medial frontal cortex is activated in advance of conscious

43 awareness of going in or out of a transient hallucinatory state. There was an increase in

44 activity initiated a few seconds before the button-press for onsets, and a corresponding

45 decrease in activity initiated a few seconds before the button-press for offsets. We identified

46 the time between onset and offset button-presses, extracted the corresponding BOLD time-

47 courses from nominated regions-of-interest, and analyzed changes in the signal from 10

48 seconds before to 15 seconds after the response-button was pressed, which identified onset

49 and offset events. We suggest that this brain region act as a switch to turn on and off a

50 hallucinatory episode. The results may have implications for new interventions for intractable

51 hallucinations. 


\section{Main text: 2661 words}

\section{Introduction}

55 Auditory verbal hallucinations (AVH) in the sense of "hearing voices" in the absence of a corresponding auditory source, is a remarkable state of the mind. AVHs were traditionally

57 seen as a hallmark of schizophrenia ${ }^{1-7}$, but also occur in other psychiatric and neurological disorders. AVH cross the border between pathological and normal states of mind, since they are experienced in about $10 \%$ of the general population ${ }^{8-11}$. As a symptom, AVHs are often experienced as highly distressing, while people in the general population are usually not

61 distressed to the same degree ${ }^{12,9}$. An equally remarkable characteristic of AVHs is that they are spontaneous episodes for which there are no known environmental triggers, occurring in

63 resting and relaxed as well as in stressful and noisy environments ${ }^{13,14}$. The same is true for the

64 offset of an episode, which likewise can occur in a variety of environmental situations. The

65 absence of environmental causes for these transient on- and off-fluctuations of AVHs would

66 thus point to an internal, i.e. some kind of neural switching mechanism, not only for the

67 spontaneous onset, but also for the spontaneous offset of an episode. We therefore studied the neural underpinnings of the spontaneous switching between AVH on- and off-states by monitoring neural activity a few seconds before and after reported onset (start) and offset

70 (stop) of a hallucinatory episode, and related this to the corresponding conscious awareness of

71 the event. Brain activity can be monitored on-line with functional magnetic resonance

72 imaging (fMRI), where changes in neural activity are estimated from modelling of the blood-

73 oxygenation-dependent (BOLD) function ${ }^{15}$. Yet this requires participants to lay still in the

74 scanner while experiencing AVH and indicating their on and offset by button-press, a very

75 demanding task (see ${ }^{9,16-19}$ ). This paradigm requires patients to be aware and thoughtful of

76 their experiences and to experience a required minimum of episodes of AVH during the

77 scanning session, as neither continuous hallucinations nor a period with too few 
78 hallucinations will allow the study of on- and offsets. Only few research groups around the

79 world have succeeded to obtain a number of such scans, typically less than 20. In order to

80 increase power to detect subtle changes in activity during brief moments of on and offsets, we

81 joined forces from three research groups to recruit a reasonably large sample of individuals

82 who were hallucinating frequently, but not continuously. The aim of the present study was to

83 use fMRI to monitor changes in neural activity on a second-by-second-basis, in a resting-state

84 situation, where subjects signaled the onset of a hallucinatory episode by pressing one

85 response-button and the offset of an episode by pressing another response-button, in the

86 course of the scanning session. A time-window was set from 10 seconds before to 15 seconds

87 after the subject had pressed a button, from which voxel-wise data were extracted, analyzed

88 and displayed second-by-second in a sliding window over the evaluation period (see

89 Methods). Data were pooled from three different sites at the University of Bergen, Norway,

90 Groningen University Medical Center, Netherlands, and Medical University of Plovdiv,

91 Bulgaria, making up a total of 66 subjects hallucinating during fMRI recordings. As

92 hallucinatory experiences cross the border between abnormal and normal conditions, we

93 included both clinical and non-clinical "voice-hearers", focusing on tracking the neural

94 signatures of AVH-experiences per se, not restricted to a particular diagnostic group or mental

95 condition.

\section{$97 \quad$ Results}

Anticipated activity patterns during hallucinatory periods

99 Functional data that were obtained using the button-press symptom-capture paradigm ${ }^{9}$

100 revealed several statistically significant clusters of increased activity during hallucinatory

101 periods (i.e. periods after onset and before offset). These clusters included the left fronto-

102 temporal language areas (Wernicke's area in the superior temporal gyrus, and Broca's area in 
103 the inferior frontal gyrus), using a FEW-corrected significance threshold of $\mathrm{p}<.05$ (see Figure

1041 and Table 1).

105

106

Insert Figure 1 OVERLAPPING ACTIVITY about here about here

107

108 We compared the anatomical localizations of these activity with areas previously reported as

109 activated during ongoing hallucinatory episodes (see meta-analysis ${ }^{16,17}$ ). As can be seen in

110 Figure 1, the overlap of the present activity with previous reports is almost perfect, thus

111 validating that the present activity reflects neural correlates of ongoing hallucinations.

112 Analyzing activity data from each of the three sites separately, the general pattern of activity

113 remained, although statistically weaker for the Bergen cohort and reduced to trend level for

114 the Plovdiv cohort. Contrasting clinical- and non-clinical voice hearers from the Groningen

115 cohort revealed overlapping activity in all areas except for the right planum temporale (PT)

116 and right lateral superior occipital cortex.

117

118 Insert Table 1 about here

119

120

121

Differential activity in the ventro-medial frontal cortex

122 Analysis of the extracted time-courses from the nominated regions of interest (ROIs),

123 separately for onset and offset of episodes, revealed a distinct decrease in activity in the

124 intersection of the paracingulate cortex, medial frontal cortex, and the frontal pole (see Figure

125 2). The decrease had a minimum peak at time $(\mathrm{t})=3 \sec (\Delta=-158 \mathrm{iu}, \mathrm{p}=0.021 \pm \sim 0.002$,

$12695 \% \mathrm{CI}$ ) relative to the button-press response (see Figure 3). This minimum preceded the 
127 corresponding motor response from the subjects' button-press which had a peak maximum at

$128 \mathrm{t}=5$ seconds.

129

Insert Figure 2 ANATOMY OF ROI about here

131

132 The decrease in activity preceding an offset button-press contrasted to an increase in activity

133 observed in advance of an onset button-press ( $\Delta=35 \mathrm{iu}, \mathrm{p}=0.014, \pm \sim 0.0016,95 \% \mathrm{CI})$,

134 occurring at around $t=-1$ second. These results were verified in an extended time-series

135 analysis (see Figure 4) averaged across subjects, and where aberrant time-courses were

136 rejected and results iteratively updated. An HRF-model fit was thereafter applied to the data,

137 revealing a similar differential pattern for onset- and offset-events as seen in Figure 3. See

138 Methods section for further details.

139

140 Insert Figure 3 TIME-COURSE about here

141

142

143 Insert Figure 4 ITERATIVE ANALYSIS about here

145 These results were obtained using the strictest criteria for interpretation of the subjects'

146 button-press response data, and were maintained (with varying levels of significance) for

147 more liberal interpretations $(\Delta=-79 \mathrm{iu}, \mathrm{p}=0.16, \pm \sim .005,95 \% \mathrm{CI}, \Delta=36 \mathrm{iu}, \mathrm{p}=0.0024$,

$148 \pm \sim .0007,95 \%$ CI for the long-block interpretation, see Methods for explanation). ROIs in the

149 in the pre-central motor-area also exhibited significantly increased activity, with peak activity

150 at $\mathrm{t}=5$ seconds; all significances after FEW-correction and p-level set to $<.05$. 


\section{$4 D$ permutation analysis}

153 Full-volume permutation analysis across the nominated time-windows further confirmed a

154 differential direction of activity for offsets versus onsets at the ventral edge of the intersection

155 of the paracingulate cortex, medial frontal cortex and the frontal pole (see Figure 2). This

156 region exhibited significantly reduced activity $\left(\mathrm{p}=2.5 \times 10^{-8}\right)$ for offset-of-hallucination events

157 relative to onset-of-hallucination events, peaking 2 seconds after the recorded button-press

158 event, and 3 seconds before the peak of the motor activity associated with the button-press

159 itself. Additional contrasting activity, not directly attributable to the motor response, was

160 observed in the inferior frontal gyrus $\left(\mathrm{p}=1.4 \times 10^{-11}\right.$, with peak at time $\left.(\mathrm{t})=17 \mathrm{sec}\right)$, and the

161 central opercular cortex bordering on Heschl's gyrus $\left(p=4.6 \times 10^{-14}\right.$ with peak at time $(t)=19$

$162 \mathrm{sec}$ ). These regions were also identified in our functional block-analysis (see Table 1), and

163 have also been mentioned in the literature ${ }^{16,17}$ (see Table 1).

166 The subsequent permutation analysis of offset-of-hallucination events against "baseline" data

167 from random time-windows showed again significantly reduced activity $\left(\mathrm{p}=5.2 \times 10^{-7}\right)$ specific to offset-of-hallucinatory events. This analysis also revealed transient increases in activity in the anterior cingulate $\left(p=3.7 \times 10^{-6}\right)$, insula/left operculum $\left(p=9.3 \times 10^{-6}\right)$, thalamus $\left(\mathrm{p}=1.8 \times 10^{-7}\right)$ and paracingulate cortex $\left(\mathrm{p}=1.8 \times 10^{-7}\right)$. Permutation analysis with onset-of-

171 hallucinatory events against "baseline" data also revealed a slight increase in activation

172 initiated before the button-press with a peak around 1 second after the button-press event $(\mathrm{p}=$

$\left.1732.5 \times 10^{-4}\right)$. Finally, there was a large and significant increase in activity in the primary motor cortex in the pre-central gyrus $\left(\mathrm{p}=7 \times 10^{-32}\right)$, representing the button-press response per se. 
177 The finding of anticipatory neural activity in the ventro-medial frontal region preceding start

178 and end of auditory hallucinations is a novel finding, which could lead to new hypotheses

179 about excitation and in particular inhibition of a hallucinatory event, and what underlies the

180 experience of the start and stop of a "voice". Both the time-course- and permutation-analyses

181 revealed a significant brain response initiated a few seconds in advance of the subject

182 becoming consciously aware of being in or out of a hallucinatory state. This suggests that the

183 ventro-medial frontal region may be crucial in both the initiation and cessation of

184 hallucinatory episodes, and speaks to a kind of regulatory role, or switch function for this

185 region. Metaphorically, it could be thought of as this region acting like a conductor, directing

186 the orchestration of neural events that leads up to a full-blown perceptual experience of

187 "hearing a voice" in the absence of a corresponding external source and also to the cessation

188 of the perceptual experience. Brain responses in advance of conscious awareness have been

189 previously reported for error monitoring, where subjects showed reduced activity in regions

190 related to the default mode network (the "oops region") seconds before they became aware of

191 having made an erroneous response $\mathrm{e}^{20}$. The present results complement recent findings of

192 aberrant functional connectivity ${ }^{21}$ and morphological differences ${ }^{22}$ in the same brain region.

193 Garrison, et al. ${ }^{22}$ used structural MRI and found that hallucinating patients had shorter

194 paracingulate sulcus than healthy controls and also to non-hallucinating patients, and

195 suggested that this region of the brain is tuned to "reality monitoring", i.e. the ability to judge

196 whether a memory comes from an outer or inner source. This suggestion ${ }^{22}$ was based on the

197 findings reported by Buda, et al. ${ }^{23}$ that otherwise healthy individuals, born without a

198 discernible paracingulate sulcus in either hemisphere, showed impaired performance on a

199 word-pair memory/imagery task. These observations ${ }^{22,23}$ may provide important clues for

200 understanding the significance of the present findings, insofar that the ventral portion of this

201 cortical region may be crucial for how AVHs are spontaneously initiated and also why they 
202 may spontaneously and transiently disappear. In this respect our data support a previous

203 report from two patients of anticipatory neural activity to onset-signaling ${ }^{24}$. A potential

204 confounding of the results could be anticipatory attention focus on motor-responses $\left(\mathrm{cf}^{25}{ }^{25}\right.$,

205 which otherwise could affect the observed activity. As seen in the lower panels of Figure 3

206 this is probably not the case, since there was a clear peak around 5 seconds post-response

207 obtained from the pre-central motor-cortex on the left side (from right-handheld response-

208 buttons), and with 5-6 times higher response amplitude as that obtained from the ventro-

209 medial frontal cortex ${ }^{26}$. This is what one would expect considering the lag of the

210 hemodynamic response relative to a neural event, but would not expect for a peak occurring

211 at 3-4 seconds and in the ventro-medial frontal cortex, after the button-press. The decrease in

212 brain activity a few seconds before the indicated awareness of the offset of a hallucinatory

213 episode, may correspond to previous findings of frontal neurotransmitter imbalance ${ }^{27-29}$.

214 Using MR spectroscopy ( $\left.{ }^{1} \mathrm{H}-\mathrm{MRS}\right)$, Ćurčić-Blake, et al. ${ }^{30}$, van Den Heuvel, et al. ${ }^{31}$ and

215 Hugdahl, et al. ${ }^{32}$ found increased levels of glutamate in frontal regions in hallucinating

216 individuals. This is in accordance with what Jardri, et al.$^{28}$ labelled the Excitatory/Inhibitory

217 (E/I) imbalance model of auditory hallucinations, and we now suggest that the offset of a

218 hallucinatory event is mediated by temporary restoring such imbalances. Future research will

219 hopefully resolve the underlying causes at the receptor and transmitter level. The present

220 findings could also have therapeutic implications in guiding more targeted brain stimulation

221 approaches. Brain stimulation interventions is a promising approach to medication-resistant

222 hallucinations ${ }^{33,34,35}$ and targeting the the ventro-medial frontal cortex using stimulation to

223 stop the onset, or accelerate the offset of an AVH-episode could be a way to help patients overcome intractable AVHs. 


\section{References}

2281 Andreasen, N. C. \& Olsen, S. Negative v positive schizophrenia. Definition and validation. JAMA Psychiatry 39, 789-794, doi:10.1001/archpsyc.1982.04290070025006 (1982). Waters, F., Badcock, J., Michie, P. \& Maybery, M. Auditory hallucinations in schizophrenia: Intrusive thoughts and forgotten memories. Cogn. Neuropsychiatry 11, 65-83, doi:10.1080/13546800444000191 (2006).

Aleman, A. \& Larøi, F. Hallucinations: The science of idiosyncratic perception. (American Psychological Association, 2008). $R$. Early manifestations and first-contact incidence of schizophrenia in differen cultures. A preliminary report on the initial evaluation phase of the WHO collaborative study on determinants of outcome of severe mental disorders. Psychol. Med. 16, 909-928, doi:10.1017/s0033291700011910 (1986).

Ford, J. M., Morris, S. E., Hoffman, R. E., Sommer, I. E., Waters, F., McCarthy-Jones, S., Thoma, R. J., Turner, J. A., Keedy, S. K., Badcock, J. C. \& Cuthbert, B. N. Studying Hallucinations within the NIMH RDoC Framework. Schizophr. Bull. 40, 295-304, doi:10.1093/schbul/sbu011 (2014).

Ford, J. M., Dierks, T., Fisher, D. J., Herrmann, C. S., Hubl, D., Kindler, J., Koenig, T., Mathalon, D. H., Spencer, K. M., Strik, W. \& van Lutterveld, R. Neurophysiological studies of auditory verbal hallucinations. Schizophr. Bull. 38, 715723, doi:10.1093/schbul/sbs009 (2012).

Hugdahl, K. \& Sommer, I. E. Auditory verbal hallucinations in schizophrenia from a levels of explanation perspective. Schizophr. Bull. 44, 234-241, doi:10.1093/schbul/sbx142 (2018). Stiles, T. C. \& Vedul $\square$ Kjelsås, E. Prevalence of auditory verbal hallucinations in a general population: A group comparison study. Scand. J. Psychol. 56, 508-515, doi:10.1111/sjop.12236 (2015).

9 Sommer, I. E., Diederen, K. M., Blom, J.-D., Willems, A., Kushan, L., Slotema, K., Boks, M. P. M., Daalman, K., Hoek, H. W., Neggers, S. F. W. \& Kahn, R. S. Auditory verbal hallucinations predominantly activate the right inferior frontal area. Brain 131, 3169-3177, doi:10.1093/brain/awn251 (2008).

10 Sommer, I. E., Daalman, K., Rietkerk, T., Diederen, K. M., Bakker, S., Wijkstra, J. \& Boks, M. P. M. Healthy individuals with auditory verbal hallucinations; Who are they? Psychiatric assessments of a selected sample of 103 subjects. Schizophr. Bull. 36, 633-641, doi:10.1093/schbul/sbn130 (2010).

11 Larøi, F., Sommer, I. E., Blom, J. D., Fernyhough, C., ffytche, D. H., Hugdahl, K., Johns, L. C., McCarthy-Jones, S., Preti, A., Raballo, A., Slotema, C. W., Stephane, M. $\&$ Waters, F. The characteristic features of auditory verbal hallucinations in clinical and nonclinical groups: State-of-the-art overview and future directions. Schizophr. Bull. 38, 724-733, doi:10.1093/schbul/sbs061 (2012). 
12 Daalman, K., Boks, M. P. M., Diederen, K. M., de Weijer, A. D., Blom, J. D., Kahn, R. S. \& Sommer, I. E. The same or different? A phenomenological comparison of auditory verbal hallucinations in healthy and psychotic individuals. J. Clin. Psychiat 72, 320-325, doi:10.4088/jcp.09m05797yel (2011).

13 Bless, J. J., Larøi, F., Kompus, K., Kråkvik, B., Vedul-Kjelsås, E., Kalhovde, A. M. \&

275

276

277

278

279

280

$281 \quad 15$

282

283

284

285

286

287

288

289

290

291

292

293

294

295

296

297

298

299

300

301

302

303

304

305

306

307

308

309

310

311 Hugdahl, K. Do adverse life events at first onset of auditory verbal hallucinations influence subsequent voice characteristics? Results from an epidemiological study. Psychiatry Res. 261, 232-236, doi:10.1016/j.psychres.2017.12.060 (2017).

14 Delespaul, P., deVries, M. \& van Os, J. Determinants of occurrence and recovery from hallucinations in daily life. Soc. Psychiatry Psychiatr. Epidemiol. 37, 97-104, doi:10.1007/s001270200000 (2002).

15 Moonen, C. T. W. \& Bandettini, P. A. Functional MRI. (Springer-Verlag, 1999).

16 Jardri, R., Pouchet, A., Pins, D. \& Thomas, P. Cortical activations during auditory verbal hallucinations in schizophrenia: A coordinate-based meta-analysis. Am. J. Psychiatry 168, 73-81, doi:10.1176/appi.ajp.2010.09101522 (2011).

17 Kompus, K., Westerhausen, R. \& Hugdahl, K. The "paradoxical" engagement of the primary auditory cortex in patients with auditory verbal hallucinations: A metaanalysis of functional neuroimaging studies. Neuropsychologia 49, 3361-3369, doi:10.1016/j.neuropsychologia.2011.08.010 (2011).

18 Dierks, T., Linden, D. E., Jandl, M., Formisano, E., Goebel, R., Lanfermann, H. \& Singer, W. Activation of Heschl's gyrus during auditory hallucinations. Neuron 22 , 615-621, doi:10.1016/s0896-6273(00)80715-1 (1999).

19 van de Ven, V. G., Formisano, E., Röder, C. H., Prvulovic, D., Bittner, R. A., Dietz, M. G., Hubl, D., Dierks, T., Federspiel, A., Esposito, F., Di Salle, F., Jansma, B., Goebel, R. \& Linden, D. E. J. The spatiotemporal pattern of auditory cortical responses during verbal hallucinations. Neuroimage 27, 644-655, doi:10.1016/j.neuroimage.2005.04.041 (2005).

20 Eichele, T., Debener, S., Calhoun, V., Specht, K., Engel, A., Hugdahl, K., Von Cramon, D. Y. \& Ullsperger, M. Prediction of human errors by maladaptive changes in event-related brain networks. Proc. Natl. Acad. Sci. U. S. A. 105, 6173-6178, doi:10.1073/pnas.0708965105 (2008).

21 Alonso-Solís, A., Vives-Gilabert, Y., Grasa, E., Portella, M. J., Rabella, M., Sauras, R. B., Roldán, A., Núñez-Marín, F., Gómez-Ansón, B., Pérez, V., Alvarez, E. \& Corripio, I. Resting-state functional connectivity alterations in the default network of schizophrenia patients with persistent auditory verbal hallucinations. Schizophr. Res. 161, 261-268, doi:10.1016/j.schres.2014.10.047 (2015).

22 Garrison, J. R., Fernyhough, C., McCarthy-Jones, S., Simons, J. S. \& Sommer, I. E. Paracingulate sulcus morphology and hallucinations in clinical and nonclinical groups. Schizophr. Bull. 45, 733-741, doi:10.1093/schbul/sby157 (2019).

23 Buda, M., Fornito, A., Bergström, Z. M. \& Simons, J. S. A specific brain structural basis for individual differences in reality monitoring. J. Neurosci. 31, 14308-14313, doi:10.1523/jneurosci.3595-11.2011 (2011). 
31224 Shergill, S. S., Brammer, M., Amaro, E., Williams, S., Murray, R. \& Mcguire, P.

313

314

315

316

317

318

319

320

321

322

323

324

325

326

327

328

329

330

331

332

333

334

335

336

337

338

339

340

341

342

343

344

345

346

347

348

349

350

351

352

353
Temporal course of auditory hallucinations. Br. J. Psychiatry 185, 516-517, doi:10.1192/bjp.185.6.516 (2004).

25 van Lutterveld, R., Diederen, K., Schutte, M., Bakker, R., Zandbelt, B. \& Sommer, I. E. Brain correlates of auditory hallucinations: Stimulus detection is a potential confounder. Schizophr. Res. 150, 319-320, doi:10.1016/j.schres.2013.07.021 (2013).

26 Logothetis, N. K. \& Pfeuffer, J. On the nature of the BOLD fMRI contrast mechanism. Magn. Reson. Imaging 22, 1517-1531, doi:10.1016/j.mri.2004.10.018 (2004).

27 Steinmann, S., Leicht, G. \& Mulert, C. The interhemispheric miscommunication theory of auditory verbal hallucinations in schizophrenia. Int. J. Psychophysiol. 145, 83-90, doi:10.1016/j.ijpsycho.2019.02.002 (2019).

28 Jardri, R., Hugdahl, K., Hughes, M., Brunelin, J., Waters, F., Alderson-Day, B., Smailes, D., Sterzer, P., Corlett, P. R., Leptourgos, P., Debbané, M., Cachia, A. \& Denève, S. Are hallucinations due to an imbalance between excitatory and inhibitory influences on the brain? Schizophr. Bull. 42, 1124-1134, doi:10.1093/schbul/sbw075 (2016).

29 Allen, P., Sommer, I. E., Jardri, R., Eysenck, M. W. \& Hugdahl, K. Extrinsic and default mode networks in psychiatric conditions: Relationship to excitatory-inhibitory transmitter balance and early trauma. Neurosci. Biobehav. Rev. 99, 90-100, doi:10.1016/j.neubiorev.2019.02.004 (2019).

30 Ćurčić-Blake, B., Bais, L., Sibeijn-Kuiper, A., Pijnenborg, H. M., Knegtering, H., Liemburg, E. \& Aleman, A. Glutamate in dorsolateral prefrontal cortex and auditory verbal hallucinations in patients with schizophrenia: A 1H MRS study. Prog. NeuroPsychopha 78, 132-139, doi:10.1016/j.pnpbp.2017.05.020 (2017).

31 van Den Heuvel, M. P., Mandl, R. C. W., Stam, C. J., Kahn, R. S. \& Hulshoff Pol, H. E. Aberrant frontal and temporal complex network structure in schizophrenia: A graph theoretical analysis. J. Neurosci. 30, 15915-15926, doi:10.1523/jneurosci.287410.2010 (2010).

32 Hugdahl, K., Craven, A. R., Nygård, M., Løberg, E.-M., Berle, J. Ø., Johnsen, E., Kroken, R., Specht, K., Andreassen, O. A. \& Ersland, L. Glutamate as a mediating transmitter for auditory hallucinations in schizophrenia: A 1H MRS study. Schizophr. Res. 161, 252-260, doi:10.1016/j.schres.2014.11.015 (2015).

33 Stoyanov, D., Stieglitz, R.-D., Lenz, C. \& Borgwardt, S. in New developments in clinical psychology research (eds D. Stoyanov \& R-D. Stieglitz) 195-209 (Nova Science Publishers, New York, 2015).

34 Dougall, N., Maayan, N., Soares-Weiser, K., McDermott, L. M. \& McIntosh, A. Transcranial magnetic stimulation (TMS) for schizophrenia. Cochrane Database Syst. Rev., doi:10.1002/14651858.cd006081.pub2 (2015).

35 Nathou, C., Simon, G., Dollfus, S. \& Etard, O. Cortical anatomical variations and efficacy of rTMS in the treatment of auditory hallucinations. Brain Stimul. 8, 11621167, doi:10.1016/j.brs.2015.06.002 (2015). 
bioRxiv preprint doi: https://doi.org/10.1101/2020 03.17.995282; this version posted March 18, 2020. The copyright holder for this preprint (which was not certified by peer review) is the author/funder, who has granted bioRxiv a license to display the preprint in perpetuity. It is made available under aCC-BY-NC-ND 4.0 International license.

\section{Table 1}

355 Table 1 shows clusters and local maxima obtained by block analysis in the present study,

356 with corresponding activations from Jardri, et al. ${ }^{16}$ and Kompus, et al. ${ }^{17}$. Clusters in the novel

357 data are thresholded non-parametrically at $Z>4.5$; corrected cluster significance is reported,

358 thresholded at $\mathrm{p}>0.05$ and extent $>20$ voxels.

359

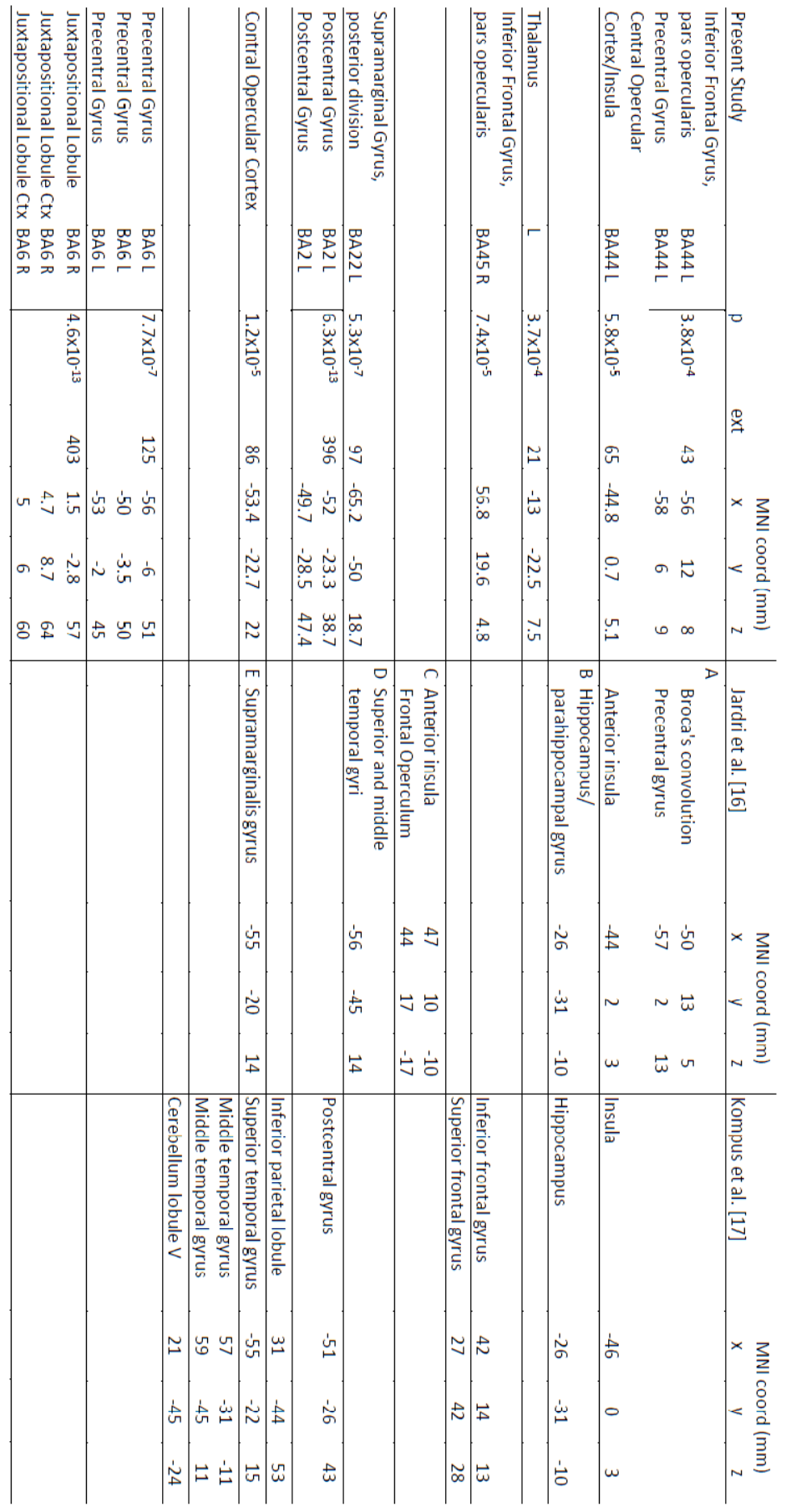


bioRxiv preprint doi: https://doi.org/10.1101/2020.03.17.995282; this version posted March 18, 2020. The copyright holder for this preprint (which was not certified by peer review) is the author/funder, who has granted bioRxiv a license to display the preprint in perpetuity. It is made available under aCC-BY-NC-ND 4.0 International license.

\section{$360 \quad$ Figure 1}

361

362 Figure 1 shows the results from the standard group-level block-analysis of the BOLD-fMRI

363 data, overlaid with peak activity from the Jardri, et al. ${ }^{16}$ and Kompus, et al. ${ }^{17}$, meta-analyses,

364 marked respectively with a blue (Jardri) and green (Kompus) 'x', verifying the presently seen

365 activity with activity previously repeatedly reported in the literature.

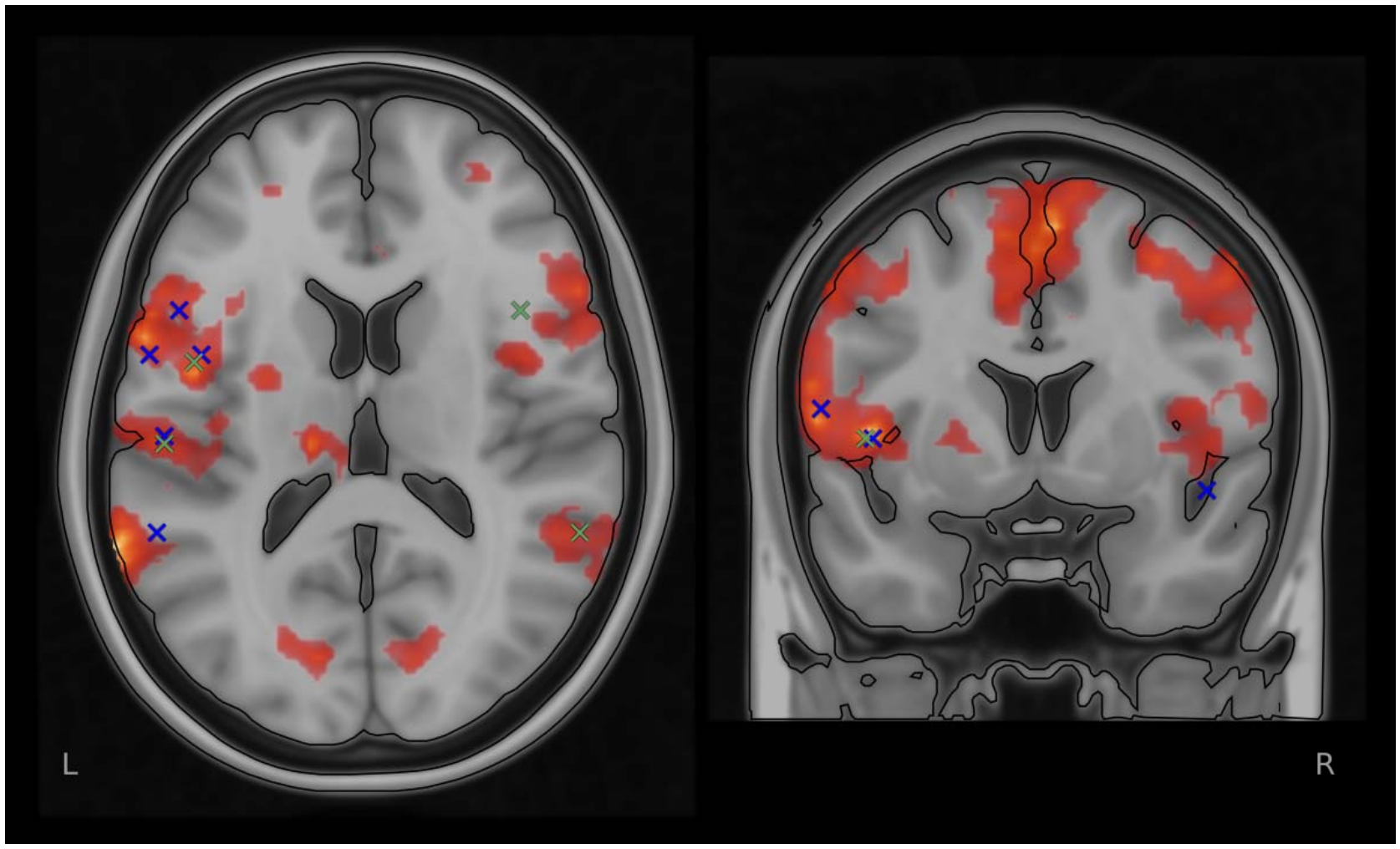


bioRxiv preprint doi: https://doi.org/10.1101/2020.03.17.995282; this version posted March 18, 2020. The copyright holder for this preprint (which was not certified by peer review) is the author/funder, who has granted bioRxiv a license to display the preprint in perpetuity. It is made available under aCC-BY-NC-ND 4.0 International license.

\section{Figure 2}

369 Figure 2 shows the anatomical localization of the ROI (turquoise) with MNI peak coordinates

$370 \mathrm{x}=8.8, \mathrm{y}=44.8, \mathrm{z}=-5.64 \mathrm{~mm}$ from where the time-courses were extracted, in the intersection of

371 the paracingulate cortex (demarcated in dark blue), medial inferior frontal cortex (demarcated

372 in green) and frontal pole (demarcated in yellow).

373

374

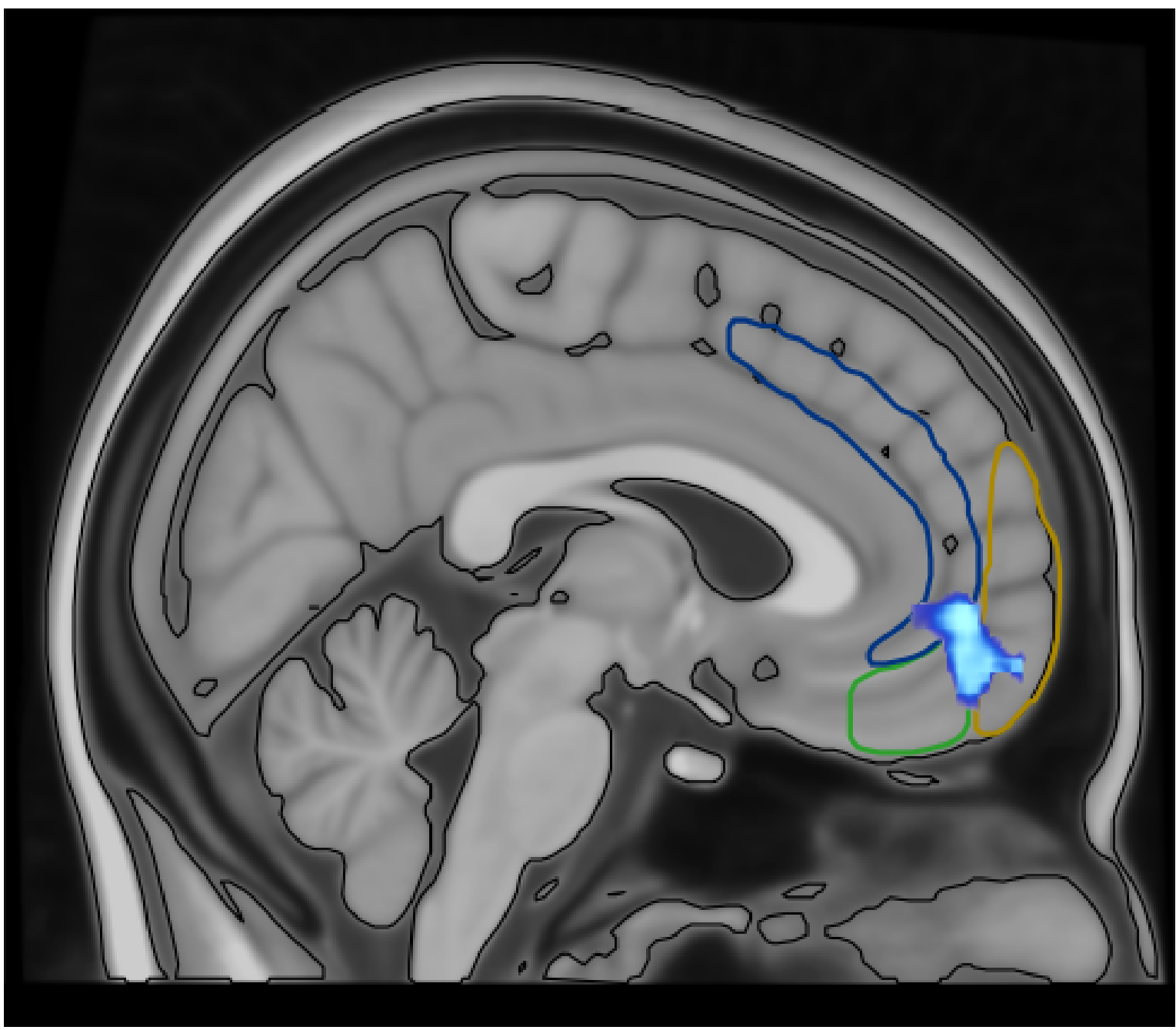




\section{Figure 3}

378 Figure 3 shows filtered time-courses tracked second-by-second around the onset-of-

379 hallucination (red) versus offset-of-hallucination (green) events from the ROI at the

380 intersection of the paracingulate cortex/medial inferior frontal cortex/frontal pole. Grey area

381 shows differential responding for onset- versus offset-events. Time " 0 " on the $\mathrm{x}$-axis represent

382 the point in time when the button-press occurred. Time -10 represent 10 secs before a button-

383 press and time 15 represents $15 \mathrm{sec}$ after a button-press. Y-axis in international units (iu). See

384 Results section for further details.

385

386

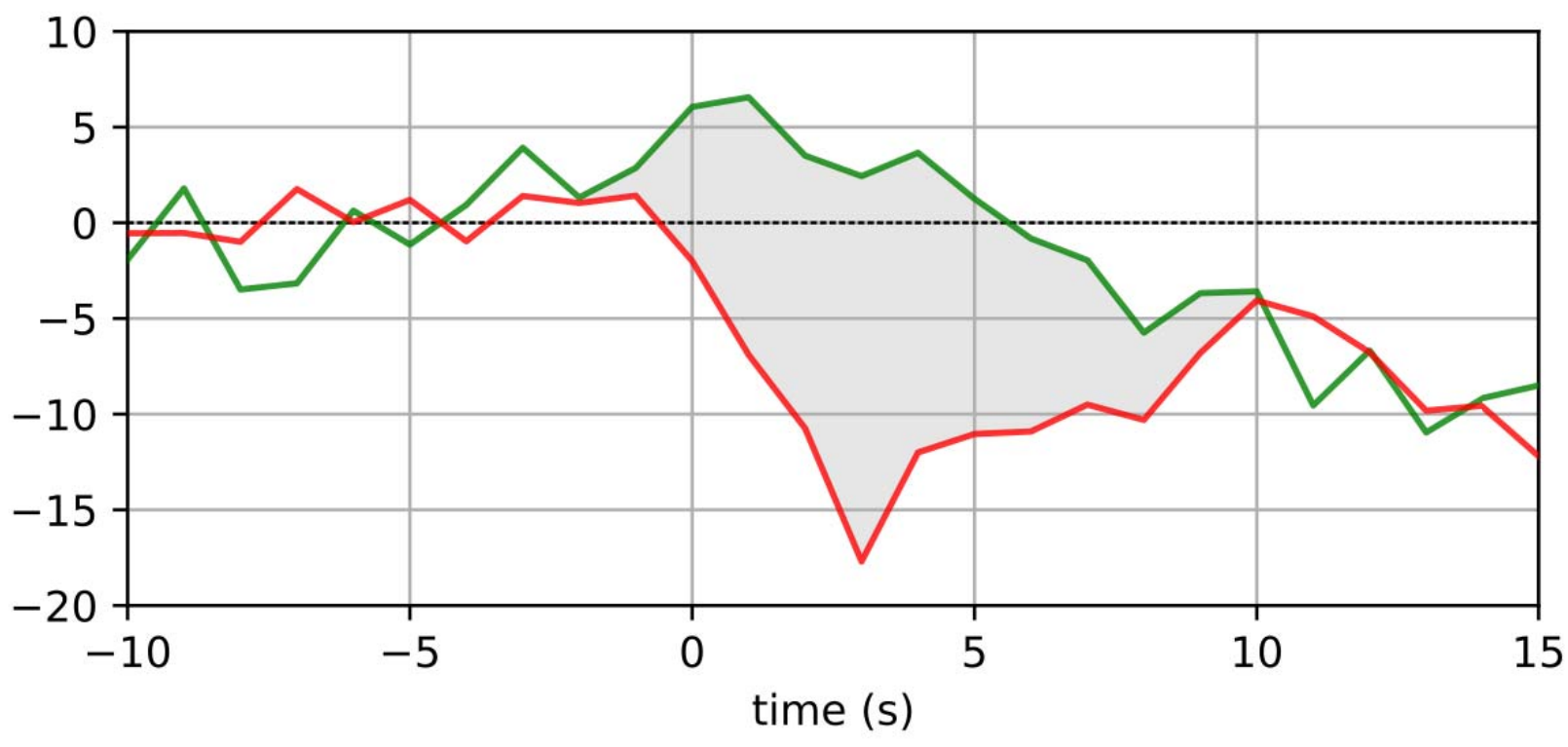




\section{Figure 4}

390 Figure 4 shows group-average (blue line) and hemodynamic response function (HRF) -

391 model fit (green line) of time-course data from the nominated ROI in the intersection of the

392 paracingulate cortex/medial inferior frontal cortex and the frontal pole, separated for onset

393 (left upper panel) and offset (left middle panel) events, for the final iteration of filtering. The

394 lower panel show corresponding time-courses for the left pre-central motor cortex (note

395 different $y$-axis scale). Adjacent panels to the right show group-mean encoded as intensity, for

396 each step (y-axis) of a leave-one-out validation according to which strongly deviant time-

397 courses were rejected. See Methods and Results sections for further details.

398
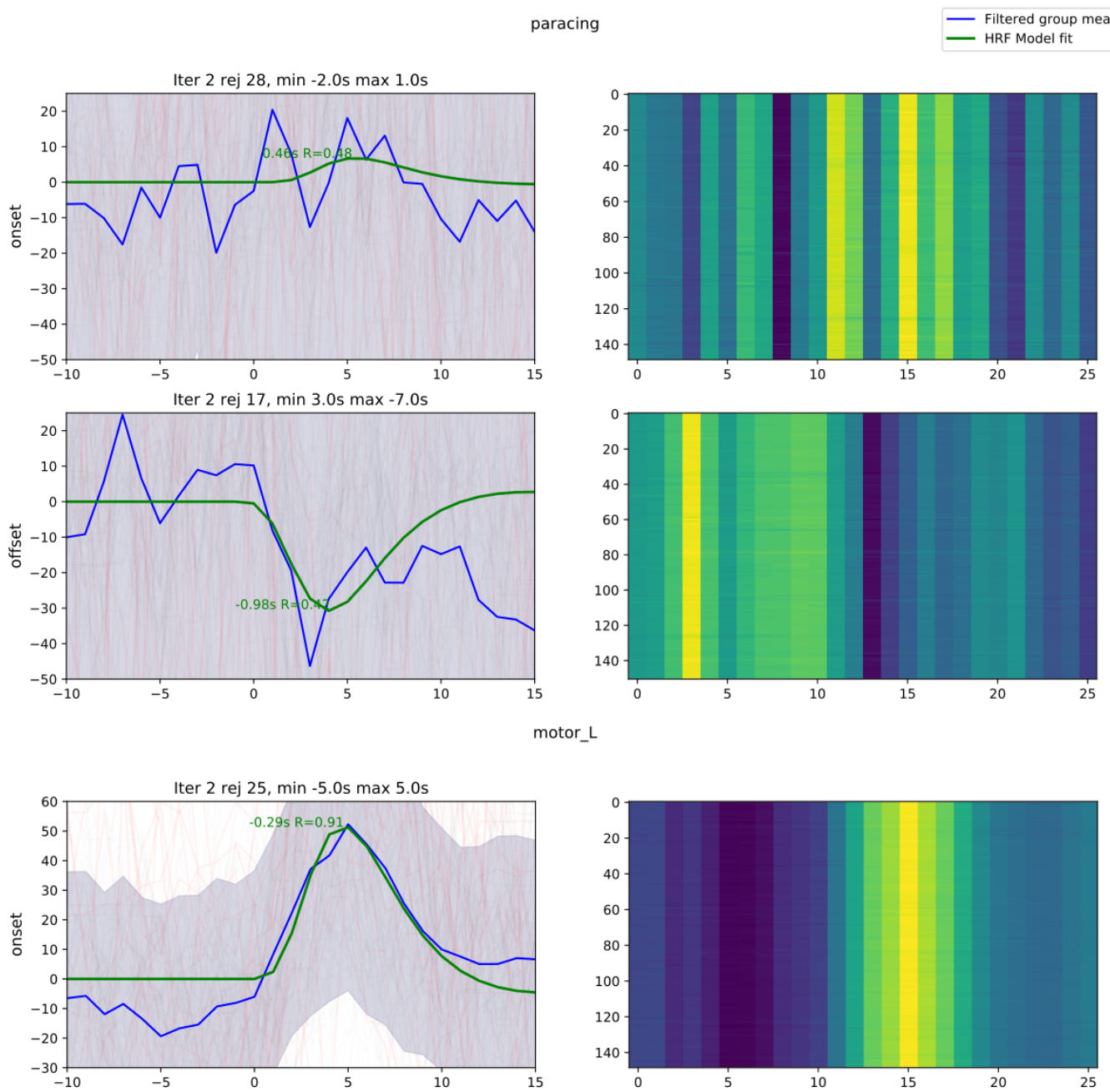
bioRxiv preprint doi: https://doi.org/10.1101/2020.03.17.995282; this version posted March 18, 2020. The copyright holder for this preprint

(which was not certified by peer review) is the author/funder, who has granted bioRxiv a license to display the preprint in perpetuity. It is made available under aCC-BY-NC-ND 4.0 International license. 


\section{$401 \quad$ Figure 5}

402 Figure 5 illustrates the three possible interpretations of subjects' response patterns. Pattern A

403 is unambiguous, and is matched identically by strict, short and long definitions. Pattern B

404 includes a spurious "offset" event; the strict definition rejects this block, the short definition

405 ends the block at the first offset, and the long definition ends the block at the last offset.

406 Pattern C shows spurious onset events; again this block was rejected by the strict definition,

407 whilst both short and long definitions match from the first "onset" event.

408

409
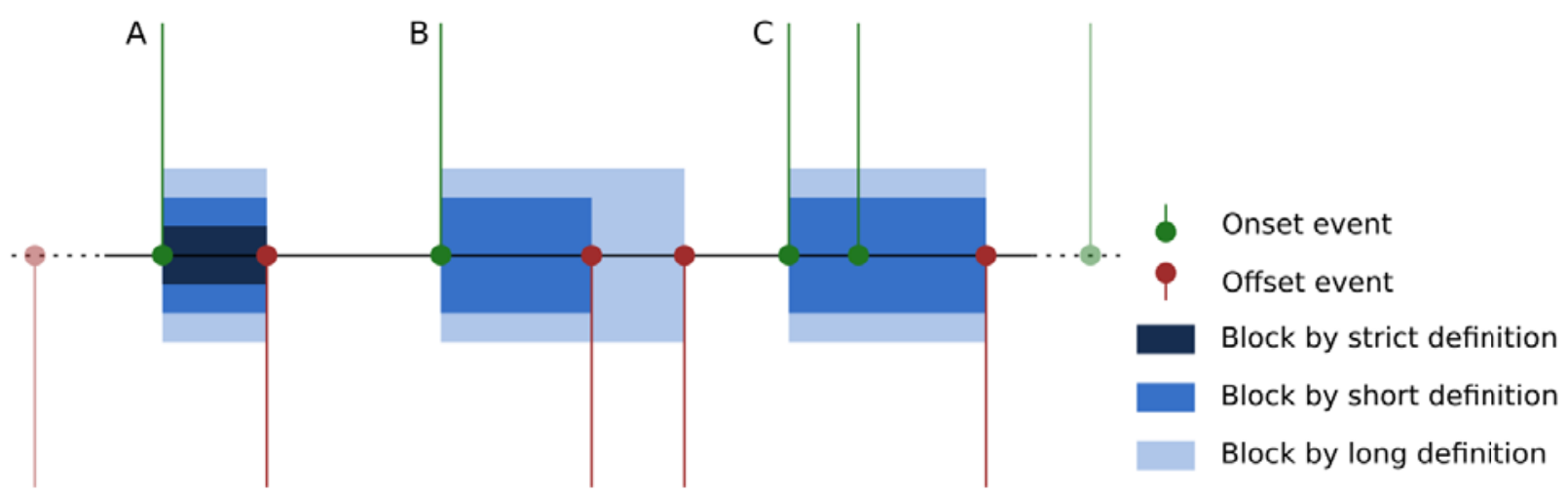

411

412 


\section{Subjects}

415 Structural data and functional BOLD data were collected from a total of 66 subjects, of which

41645 were diagnosed with an ICD-10 or DSM-IV schizophrenia spectrum disorder. The patients

417 came from three collaborating projects and sites. These were University of Bergen, Norway

$418(\mathrm{n}=11,7$ males, mean age 27.8 (SD 7.0) years); Plovdiv Medical University, Bulgaria ( $\mathrm{n}=13$,

41911 males, mean age 35.3 (SD 14.0 years), Groningen University Medical Center, Netherlands

$420(\mathrm{n}=21,7$ males, mean age 39.0 (SD 11.4) years), total 25 males and 19 females, mean age

42137.9 (SD 13.2) years. Symptom severity for patients was assessed with the positive and

422 negative syndrome scale $\left(\mathrm{PANSS}^{36}\right.$ ). Mean total PANSS score was 64.9 (SD 16.9). In order to

423 be included in the study, patients had to score $>3$ on the PANSS, P3 hallucinatory behavior

424 item within a week of the MR scanning (mean P3 score 4.6 (SD 1.1)). The patients were all

425 on second-generation antipsychotics (often clozapine), with some patients in addition being

426 prescribed antidepressants and/or anxiolytics. Mean antipsychotic Defined Daily Doses

427 (DDD) were 1.228 (SD 0.578). The total sample also consisted of 21 non-clinical

428 hallucinating individuals (4 males, mean age 44.5 (SD 13.0) years), i.e. in whom a clinical

429 axis I and axis II diagnosis was ruled out using the CASH and SCID-II interview, included in

430 the Groningen University Medical Center sample (for details see ${ }^{10}$ ). This yielded a total

431 sample of 29 males, 37 females, mean age 38.2 (SD 13.0) years. The study was approved by

432 the local ethics committees at each site, and had a European Research Council Ethics

433 Approval (ERCEA 2016-439428). Transfer of data between the sites and re-analysis at the

434 Bergen University was approved by the ethical committees at each site, and further confirmed

435 by the Regional Committee for Medical Research Ethics in Western Norway (REK-Vest

$436 \quad \# 2017 / 933)$. 


\section{Data collection}

439 Functional MR data were collected with a "symptom-capture" paradigm", where subjects

440 were instructed to press a button when a hallucinatory episode began (onset), and to press

441 another button when the episode ended (offset). The instruction of when to press the buttons

442 was presented visually through LCD goggles mounted on the head-coil, in the language

443 appropriate to the location, along with a fixation cross that was displayed in the middle of the

444 visual field. A time-window was set from 10 seconds before to 15 seconds after the subject

445 had pressed a button, from which voxel-wise data were extracted, analyzed and displayed in a

446 sliding window over the evaluation period. Particulars of the acquisition varied between sites.

447 Gradient Echo Planar Imaging (EPI) was used to collect functional BOLD data on all sites.

448 Data from Bergen were collected using a 3T GE 750 scanner with a 32-, or 8-channel head

449 coil (300 volumes at $\mathrm{TR}=2000 \mathrm{~ms}$, total duration $10 \mathrm{~min}, \mathrm{TE}=30 \mathrm{~ms}$, flip angle $90^{\circ}$,

450 resolution $128 \times 128$, pixel spacing $1.72 \mathrm{~mm}, 30$ or 26 slices of $3 \mathrm{~mm}$ thickness with $0.5 \mathrm{~mm}$

451 gap). Plovdiv data were collected with a 3T GE 750w scanner and 24-channel head coil (900

452 volumes at $\mathrm{TR}=2000 \mathrm{~ms}$ (total duration $30 \mathrm{~min}$ ), $\mathrm{TE}=30 \mathrm{~ms}$, flip angle $90^{\circ}$, resolution $64 \mathrm{x}$

45364 , pixel spacing $3.44 \mathrm{~mm}, 34$ slices of $3 \mathrm{~mm}$ thickness with $0.5 \mathrm{~mm}$ gap). Groningen data

454 were acquired on a 3T Philips Achieva scanner, as 800 volumes at TR $=21.75 \mathrm{~ms}$ (total

455 duration $8 \mathrm{~min}, 6 \mathrm{sec}), \mathrm{TE}=32.4 \mathrm{~ms}, 64 \times 64,4 \mathrm{~mm}$ voxel size, 40 slices ( $4 \mathrm{~mm}$ thickness),

456 no gap. This scan sequence achieves full brain coverage within 609 ms by combining a 3D-

457 PRESTO pulse sequence with parallel imaging (SENSE) in two directions using a

458 commercial 8-channel SENSE head-coil. A high-resolution structural T1 volume was

459 acquired for each subject, along with additional sequences that varied between sites, of no

460 relevance and are not reported herein.

461

462 Motor (button-press) response data 
463 Upon visual inspection of the subjects' button-press data, it was found that a certain number

464 of subjects reported multiple episode-onsets which did not distinctly match to a single end-of-

465 "voice" event, which required interpretation and operational definition of episodes. We

466 interpreted and operationally defined the relationship between onset- and offset button-

467 responses in three different ways, described and illustrated in Figure 5 below. According to

468 each definition, subjects' button-press response-data were filtered to remove spurious

469 episodes, and extract distinct blocks of hallucinatory vs non-hallucinatory periods. Additional

470 criteria regarding minimal spacing between events ensured the validity of the subsequent

471 analyses.

472

Operational definition of hallucinatory and non-hallucinatory periods

474 According to a first possible interpretation and definition, denoted "short blocks",

475 hallucinatory periods were defined as spanning from the moment a subject first pressed the

476 button indicating the start-of-voice episode, until they first pressed the button to indicate the

477 end-of-voice episode. This yielded 1055 usable blocks across all subjects. In a second

478 interpretation, denoted "long blocks", hallucinatory periods were defined as spanning from

479 the moment a subject first pressed the button indicating the start-of-voice episode, until the

480 last press indicating the end-of-voice episode before the next reported start-of-voice episode.

481 This yielded 1000 usable blocks across all subjects. The final, "strict blocks" interpretation,

482 accepted only periods unambiguously bounded by a single start-of-voice and a single end-of-

483 voice button-press. This yielded 300 usable blocks across all subjects. Figure 5 shows the

484 different interpretations, illustrated on a representative subject's response data. 


\section{Pre-processing of fMRI data}

489 Functional MR data were pre-processed using standard tools from FSL 5.0.11 (FEAT

490 pipeline), with additional filtering for artifacts using the ICA-AROMA method ${ }^{37-40}$. Brain-

491 masks for each subject's structural T1-images and functional data were derived using FSL's

492 bet 2 tool, with fractional intensity threshold and initial mesh center-of-gravity tuned on a

493 group basis to accommodate differences between the three sites. Individual brain-masks were

494 manually subject to visual quality control to ensure completeness and specificity of the mask.

495 When necessary, brain masking was repeated for a small number of subjects using

496 individually tuned parameters. Next, individual brain-extracted functional data were subject to

497 motion correction, using FSL's MCFLIRT utility ${ }^{38,41}$. Data were filtered spatially with a $5 \mathrm{~mm}$

498 FWHM Gaussian kernel, and temporally with a $100 \mathrm{sec}$ high-pass filter. They were then

499 registered to individual, brain-extracted high-resolution structural images using FSL's linear

500 registration tool (FLIRT), with the recommended Boundary-Based Registration mode and a

501 restricted (35 degree) search range. Registration was inspected visually for validity; for three

502 subjects where the BBR method performed poorly, a 12-degree-of-freedom registration was

503 substituted. Individual brain-extracted high-resolution structural images were registered to a

504 standard 2mm T1 template in MNI152 space (ICBM152, non-linear, $6^{\text {th }}$ generation), with a

505 12-degrees-of-freedom linear registration using FSL FLIRT, followed by a nonlinear

506 registration with $10 \mathrm{~mm}$ warp resolution using FSL FNIRT. The ICA-AROMA method was

507 applied to filtered, native-space functional data to remove residual signals associated with

508 motion artifacts and noise. Finally, per-subject native-space functional masks were

509 transformed into standard space using non-linear parameters derived from the registration

510 steps; these masks were assessed programmatically to ensure adequate coverage of the brain,

511 with particular attention to prescribed regions of interest (ROIs), see below. 


\section{Block analysis}

514 To confirm the validity of the filtered response data, a standard fMRI block--analysis was

515 performed using FSL FEAT first-level and higher-level analysis pipelines ${ }^{42}$, applied for the

516 long block definition. Mixed effects modeling (FLAME 1+2) was used for higher-level

517 analysis; clusters were thresholded non-parametrically at Z>4.5; corrected cluster significance

518 thresholded at $\mathrm{p}>0.05$ and extent $>20$ voxels.

519

520 Time-course analysis

521 For each hallucinatory period, identified as demarcating the start or end of a hallucinatory episode, windows of functional data were extracted extending from a time of $\mathrm{t}=-10 \mathrm{sec}$ through to $t=+15 \mathrm{sec}$, relative to the moment in time at which the button-press event occurred (set to $t=0 \mathrm{sec}$ ), as a continuous variable. Since the temporal resolution of functional data is relatively coarse, and also varied between sites in the current dataset, data were sampled at regular $1 \mathrm{sec}$ intervals, weighted and normalized according to a Gaussian kernel in the temporal dimension $(\mathrm{FWHM}=0.94 \mathrm{sec})$. An initial principal component analysis (PCA) on grouped, extracted functional segments, guided the selection of ROIs for further inspection and analysis. Cluster locations identified in the functional analysis and

530 those nominated and reported in previous meta-analyses ${ }^{16,17}$ were also inspected for overlaps 531 of activity between the current study and activity reported in the meta-analyses (see Figure 1

532 and Table 1). For each nominated ROI, and for each of the extracted functional segments,

533 time-course vectors were obtained and spatially averaged over a $5 \mathrm{~mm}$ radius sphere,

534 allowing activity in each region to be examined and evaluated in the time-frame leading up to and immediately following a button-press, marking the onset and offset of a hallucinatory

536 episode. Separately for onset and offset events, time-course vectors for each region were

537 aligned in the temporal dimension to the group-average for the respective region (maximizing 
538 cross-correlation). Aberrant time-courses (correlation varying by more than two standard

539 deviations) were rejected, iteratively updating the group to refine an estimated model time-

540 course for the region around onset and offset events as shown in Figure 4. A dual-gamma

541 hemodynamic response-function (HRF) model was thereafter fitted to the refined time-course

542 model, allowing magnitude and timing of any activity-related peak to be identified and

543 statistically evaluated. Random permutation-analysis $(n=5000)$ was performed to identify

544 differential effects on fit parameters between onset- and offset-events.

545

546 4D permutation analysis

547 The ROI-based analysis was generalized to a full four-dimensional (4D) permutation analysis,

548 characterizing activity specific to onset- or offset-events at each time-point in the extracted

549 window segments, searching across the entire brain volume voxel-wise. Due to the large

550 volume of data and computationally intensive nature of the permutation analysis, it was

551 necessary to develop a new software tool to facilitate this analysis. P-values were extracted (n

$552=10,000$ permutations), along with $\mathrm{p}$-values calculated on a gamma approximation of the

553 obtained distribution ${ }^{43}$, for each voxel, at each time-point. Initially, time-windows associated

554 with onset- and offset-events were contrasted jointly in the permutation analysis, yielding

555 differential effects for onset and offset events. Subsequently, time-points from offset- and

556 onset-events were separately contrasted against windows extracted around random time-

557 points (without synchronization to subjects' button-responses), as a baseline state.

Methods references:

$56036 \quad$ Kay, S. R., Fiszbein, A. \& Opler, L. A. The positive and negative syndrome scale (PANSS) for schizophrenia. Schizophr. Bull. 13, 261-276, doi:10.1093/schbul/13.2.261 (1987). 
56337 Pruim, R. H. R., Mennes, M., van Rooij, D., Llera, A., Buitelaar, J. K. \& Beckmann,

564

565

566

567

568

569

570

571

572

573

574

575

576

577

578

579

580

581

582

583

584

585

586

587

588

589

590

591

592

593

594

595

596

597 C. F. ICA-AROMA: A robust ICA-based strategy for removing motion artifacts from fMRI data. Neuroimage 112, 267-277, doi:10.1016/j.neuroimage.2015.02.064 (2015).

38 Jenkinson, M. \& Smith, S. A global optimisation method for robust affine registration of brain images. Med. Image Anal. 5, 143-156, doi:10.1016/S1361-8415(01)00036-6 (2001).

39 Woolrich, M. W., Ripley, B. D., Brady, M. \& Smith, S. M. Temporal autocorrelation in univariate linear modeling of fMRI data. Neuroimage 14, 1370-1386, doi:10.1006/nimg.2001.0931 (2001).

40 Jenkinson, M., Beckmann, C. F., Behrens, T. E. J., Woolrich, M. W. \& Smith, S. M. FSL. Neuroimage 62, 782-790, doi:10.1016/j.neuroimage.2011.09.015 (2012).

41 Jenkinson, M., Bannister, P., Brady, M. \& Smith, S. Improved optimization for the robust and accurate linear registration and motion correction of brain images. Neuroimage 17, 825-841, doi:10.1006/nimg.2002.1132 (2002).

42 Woolrich, M. W., Behrens, T. E. J., Beckmann, C. F., Jenkinson, M. \& Smith, S. M. Multilevel linear modelling for fMRI group analysis using Bayesian inference. Neuroimage 21, 1732-1747, doi:10.1016/j.neuroimage.2003.12.023 (2004).

43 Winkler, A. M., Ridgway, G. R., Douaud, G., Nichols, T. E. \& Smith, S. M. Faster permutation inference in brain imaging. Neuroimage 141, 502-516, doi:10.1016/j.neuroimage.2016.05.068 (2016).

\section{Data availability statement}

The datasets analysed during the current study are not publicly available due to restrictions imposed by Regional Committee for Medical Research Ethics in Western Norway (REK Vest) and the Data Protection Officer of the Western Norway Health Authorities

(Personvernombudet) but are available from the corresponding author on reasonable request

\section{Software/Code availability statement}

Novel in-house developed software implemented for this study has been made publicly available here: https://git.app.uib.no/bergen-fmri/functional-transients. All other stages of analysis were performed using widely-available open-source software, including tools from the FSL suite and additional filtering with the ICA-AROMA method.

\section{Author contributions}

KH designed the study, participated in patient recruitment, data analysis and interpretation, wrote the ms, ARC analyzed the data, participated in data acquisition and interpretation, 
598 commented on the ms, EJ participated in patient recruitment, commented on the ms, LE

599 participated in data acquisition and analysis, commented on the ms, DS participated in patient

600 recruitment. commented on the ms, SK participated in patient recruitment, commented on the

$601 \mathrm{~ms}$, LBS participated in organization of data and ms and commented on the ms, RAK

602 participated in patient recruitment, commented on the ms, EML participated in patient

603 recruitment, commented on the ms, IES participated in patient and subjects recruitment, data

604 interpretation, and commented on the ms.

605 Supplementary information

606 Correspondence and requests for materials should be addressed to Kenneth Hugdahl

607 (hugdahl@uib.no).

608 Competing interests

609 The co-authors Kenneth Hugdahl, Alexander R. Craven and lars Ersland own shares in the

610 company NordicNeuroLab, Inc. (https://nordicneurolab.com/) that produced add-on

611 equipment used for BOLD-fMRI data acquisition. All authors declare no conflict of interest.

\section{Acknowledgments}

613 The authors want to acknowledge the contribution by MR-technicians and participants for

614 making the study possible.

615 